\title{
CARTOX Grade 2
}

National Cancer Institute

\section{Source}

National Cancer Institute. CART OX Grade 2. NCI Thesaurus. Code C155297.

A score of 3-6 on the CART OX-10 assessment, indicating moderate neurotoxicity. 